\title{
Agent-supported Cooperative Learning Environments
}

\author{
Anton Nijholt \\ Centre of Telematics and Information Technology (CTIT) \\ PO Box 217, 7500 AE Enschede, the Netherlands \\ anijholt@cs.utwente.nl
}

\begin{abstract}
We survey our research on (3D) virtual environments inhabited by agents that help visitors to get information and to get a task done. The main ideas and designs can be tuned to different applications, including information and transaction services (e-commerce), collaborative work and educational domains.
\end{abstract}

\section{Introduction}

Virtual worlds provide interesting and different potential as learning environments. We can have virtual classrooms, which allow students to communicate with the teacher and other students. Such classrooms allow the teacher to present her courses and exercises using multimedia presentations and they allow the students to explore the presentations and, using links and annotations provided by teacher and other students, course related material. Teacher and students may have on-line sessions, presenting and discussing the topics and the organization of the course. Even these sessions can become part of the material available to students.

This approach to the learning environment may lead to enhanced environments for computer supported cooperative learning. The learning environment is distributed. Both students and teachers can add to the environment. We discuss a 3D virtual environment that has similar properties. The environment invites visitors to explore its stored and visualized information. The domain is a theatre information and transaction environment. Not only the theatre, but also the agents that communicate with the visitors have been visualized. The environment has been built using a 3D modeling language. Visitors can 'walk' from one location to the other, they can see 'information', they can explore visualized information and they can discuss information with domain agents (e.g., assistants, guides, teachers) and with other visitors (students, teachers, etc.).
In [2] commercially available virtual environments and the possibility to use them as a teaching environment have been investigated. Our claim, based on our experiences reported here, is that it is profitable to design and implement teaching environments that are tuned to particular tasks and domains. As we will illustrate, it is necessary that standards become available in order to make it possible to share environments, scenes and agents, to allow communication between agents and to obtain portable domain, instruction and student models.

\section{The Virtual Environment}

Our virtual theatre environment was built (in VRML) according to design drawings of the architects of an existing theatre [4]. Visitors can explore the environment, walk around, ask questions to visible agents, click on objects, etc. Karin, the $3 \mathrm{D}$ receptionist, is an embodied agent that understands natural language input and uses a text-to-speech system synchronized with lip movements. She has extensive knowledge of performances. Other agents include a navigation agent. It knows about the building and can be addressed using speech. A Java framework provides the protocol for communication between agents. Current research on the navigation agent deals with user preferences for navigation, modeling navigation knowledge and dialogues, adding instruction models to agents and visualization.

\section{Agents that Assist and Instruct}

Although Karin and the navigation agent assist the visitor, they have not been designed with an explicit teaching goal in mind. An other agent, called Jacob, is in development and it is meant to perform different instruction tasks. One may ask why our environment needs an agent that instructs visitors. From our point of view the most obvious answer is that it is worthwhile to learn about our environment, it is worthwhile to explore its possibilities, to discover which other visitors are there, their interests 
and their willingness to share knowledge and information, etc. Rather than having an environment to learn about the real world, we can ask students to develop skills to deal with virtual environments.

In our environment the visitor can be taught about the exploration of the environment, the knowledge that is available and the underlying databases. Presently research focuses on the abilities of the navigation agent and the use of agents that have been defined in general settings of a domain, an instruction and a student model.

\section{The Jacob Instruction Agent}

Jacob is an animated agent that gives instruction and assistance for tasks that a student has to learn to perform in virtual reality. The use of a lifelike agent in an interactive learning environment has a strong positive impact on students [4]. The interaction between students and Jacob is multimodal, through keyboard natural language, actions, and manipulations of objects. Other issues in the Jacob project are the integration of natural language dialogue in virtual reality, interactive aspects of virtual reality, and software engineering aspects. In a prototype version Jacob instructs the user in the Towers of Hanoi game (Fig. 1). We are developing the system in a generic way using Java and VRML.

Jacob involves integration of research in intelligent tutoring systems ([1,5]), graphics, AI and multi-agents, 3D visualization, and NL understanding. Fig. 2 shows the architecture of the system. Jacob has been designed in accordance with the H-Anim standard. Therefore it can be used in any VRML environment and it can be given a different task and instruction model.

\section{Domain and Alter Ego Agents}

The agent framework allows us to introduce a multitude of potential and useful agents, where some just perform some animation, others can walk around (e.g., this would be useful for a navigation agent) and others have some built-

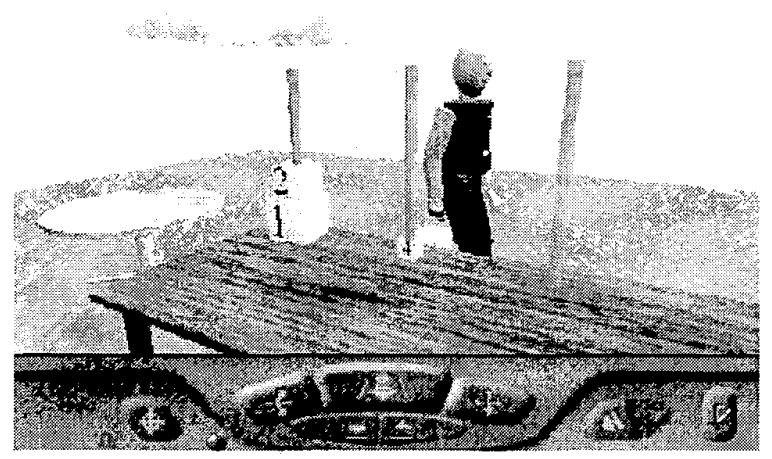

Figure 1: Jacob demonstrating the Towers of Hanoi

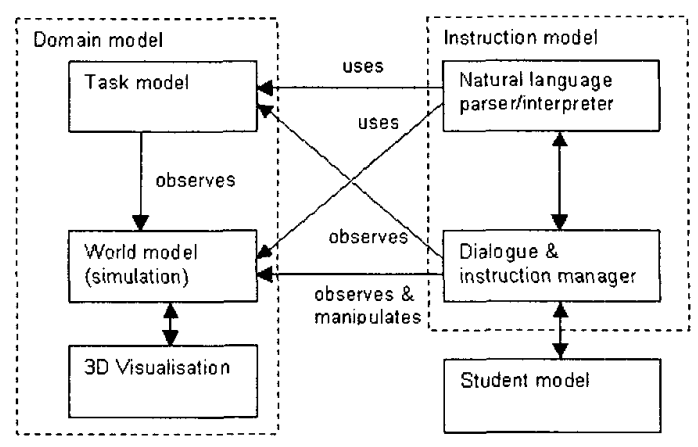

Figure 2: Software architecture of the Jacob system

in intelligence that allows them to execute certain actions based on interactions with visitors. Moreover, we embedded our environment in a multi-user shell, which means that visitors become visible as avatars (VRML objects) to which we can assign animations, but also intelligence and interaction abilities which can reflect those of the visitor, but not necessarily, since we can modify them to suit our purposes. E.g., we can have user profiles assigned by the system to a visitor's avatar acting in the virtual environment. In this way we can anticipate different behavior by reading the visitor's profile.

\section{Conclusions}

In order to maintain a virtual environment where we have a multitude of domain and user-defined agents we need some uniformity from which we can diverge in several directions and combinations of directions: agent intelligence, agent interaction capabilities, agent visualization and agent animation. Standards allow that applications can be assembled from components developed independently by multiple suppliers. Standards will also make it possible to use task and instruction models that have been defined elsewhere and tune them to a new application.

\section{References}

[1] J. Bonar et al., "An Object-Oriented Architecture for Intelligent Tutoring Systems". Proc. OOPSLA '86, 269-276.

[2] M.D. Dickey, 3D Virtual Worlds and Learning. Ph.D. Thesis, The Ohio State University, 1999.

[3] J.C. Lester et al., "The persona effect: affective impact of animated pedagogical agents". Proc. CHI'97, 359-366.

[4] A. Nijholt et al., "Multimodal Interactions with Agents in Virtual Worlds". In: Future Directions for Intelligent Systems and Information Science. N. Kasabov (ed.), Springer, 2000.

[5] B. Tekinerdogan et al., "Design of a Modular Composable Tutoring Shell for Imperative Programming Languages". Proc. Int. Conf. on Computers in Education, 1995, 356-363. 